\section{Reproductive health care in Tanzania}

\section{Introduction}

Tanzania is located on the eastern coast of Africa below the Equator, and comprises the mainland and Zanzibar Island, the latter having two parts, Unguja and Pemba. In common with many other developing countries, Tanzania is faced by multiple social and economic problems, including rapid population growth. The Bureau of Census estimates that by 2025, Tanzania's population will increase by approximately $60 \%$. Unfortunately this change is not matched by economic growth.

Although the Population Bureau reports that between 1980 and 2005 the fertility rate (i.e. the average number of children that a woman gives birth to in her lifetime) has dropped from 6.5 to 5.3 , it is still high given the resource-poor setting. The Reproductive and Child Health Department of the Ministry of Health has made great efforts to ensure family planning and child health services are available, accessible and affordable to all areas including the underserved communities. Through the national family planning programme, the Ministry of Health has worked closely with international organisations and non-governmental organisations such as EngenderHealth and other faith-based organisations. These efforts have proved effective, as the prevalence of contraceptive use increased from $10 \%$ in 1991 to $25 \%$ in 2004. However, progress is considered to be slow when compared with neighbouring countries like Kenya and Zimbabwe.

The commonly used contraceptive methods in Tanzania are oral contraceptives and injectables. There is very low use of permanent surgical methods, especially vasectomy, and other methods like spermicides. Many factors could be contributing to this scenario, including inadequate dissemination of information to clients, unavailability of the service, and unskilled personnel. The following three recently published articles describe some of the challenges of reproductive health care provision in Tanzania.

Knowledge, attitude and acceptability of spermicidal contraception among university students in Dar es Salaam, Tanzania. Mwambete K. Mogasa C. East Afr J Public Health 2008; 4: 23-27

Mwambete and Mogasa report on a study that aimed to assess the knowledge, attitudes and acceptability of spermicidal contraception amongst university students in Dar es Salaam. This topic is very important since spermicides appear to be a forgotten method of contraception (oral and injectable forms of contraception are the most popular methods). Furthermore, some of the spermicides such as nonoxynol-9 (N-9) are said to have a dual action of contraception, also killing HIV and other STI pathogens. The study was a cross-sectional questionnaire study involving 300 students drawn from the three university campuses. The findings show that the majority of the students had heard about the spermicidal contraceptive method, however they had very poor knowledge of its benefits. In addition, the actual number of users of the method was very low, accounting for $0.7 \%$ of all contraceptive methods. The authors asked students the reasons why they did not use spermicides. The majority responded that it was due to lack of familiarity with the method, although some had simply opted for other methods. Some of the students felt that the spermicides were expensive, and a small percentage feared the possibility of developing cancer with their use. The lesson we learn from such a study is the importance of raising awareness in the community and academic institutions about different contraceptive methods and of ensuring accessibility of the service. Interestingly, one of the campuses was a medical school and even the medical students demonstrated poor knowledge of this method.

Factors affecting vasectomy acceptability in Tanzania. Bunce A, Guest G, Searing H, et al. Int Fam Plann Persp 2007; 33: 13-21

Bunce et al. report on a qualitative study they performed on the factors affecting vasectomy acceptability in Kigoma, Tanzania. The approach was through focus group discussions and indepth interviews with potential and actual sterilisation clients and their partners, addressing the vasectomy decision-making process. The analysis of the discussion generated six influences on decision-making: economic hardship; spousal influence; religion; provide reputation and availability; uncertainty about the future; and poor vasectomy knowledge and understanding. This study highlighted the fact that spousal discussion was important in the decision to have a vasectomy but that such discussion needed to be initiated by the male partner. This suggests that more effort should be made to ensure that male partners have knowledge of contraceptive options and are involved in reproductive health discussions. A number of issues also arose from the discussions about religious barriers to vasectomy. For example, Roman Catholics believe that family planning methods such as vasectomy and pills do cause cancer, while the Seventh Day Adventists are strong advocates of family planning. The authors clearly outline several limitations to the study and caution readers that the findings obtained from this small, qualitative study canno necessarily be generalised to other settings. I would also be interesting to ascertain views about acceptability of vasectomy among other men and women who are neither potential, nor actual, sterilisation clients.

Motherhood status and union formation in Moshi, Tanzania 2002-2003. Hattori MK, Larsen U. Popul Stud (Camb) 2007; 61: 185-199

Hattori and Larsen assessed the effect of a premarital first birth on entrance into a first union in the Moshi urban area of Tanzania. The data were obtained from the Moshi Household Infertility Survey of 2002-2003, in which 2019 women in the reproductive age group of 20-44 years were interviewed. It has been noted previously that there has been an increase in age at first union in sub-Saharan Africa. Many studies have shown that the high level of education and urbanisation could be contributing factors Women from such backgrounds delay making a marriage commitment. However, there is concern that the increase in age of first union may result in a high rate of premarital births, as many of these women do not practise abstinence, nor do they use family planning methods, and at the same time abortion services are still illegal in most African countries. The authors found that women who spent less than a year as single mothers were significantly more likely than childless women to enter into a first union, although the magnitude of this association was weaker for more recent cohorts. The findings are supported by a previous qualitative study performed in the same area which demonstrated that men postponed paying the bride price and marriage until after the birth of a child. The authors also found that women who had been single mothers for 5 years or more (about two-thirds of women with a premarital birth) were significantly less likely than women without children to enter into a first union. These women are disadvantaged in the marriage market and as a result they end up suffering social and economic problems, since they are stigmatised and lack supportive care of their children from a partner.

\section{Conclusions}

In summary, all three studies show the urgent need for the Tanzanian Government, in collaboration with all relevant stakeholders, to strengthen family planning, starting with the local community in the underserved areas to the urban areas of Tanzania. The Government needs to ensure that the people are well informed about the different family planning options, the short- and long-term benefits, and the few side effects associated with the various methods. The next step is to ensure that the services are accessible and affordable. This is a great challenge to such a poor country like Tanzania. However, with commitment from political leaders and stakeholders, resources can be reallocated to this priority problem, with the aim of eventually improving the economy, and health, of the people and the country as a whole.

Reviewed by Miriam Mgonja, MD MMED in OB/GYN

PhD Student, Department of Public Health Sciences, University of Edinburgh, Edinburgh, UK and Obstetrician/Gynaecologist, Department of Obstetrics and Gynaecology, Muhimbili National Hospital, Dar es Salaam, Tanzania, East Africa

\section{Risk factors for arterial and venous thrombosis}

Cardiovascular risk factors and venous thromboembolism: a meta-analysis. Ageno W, Becattini C, Brighton T, Selby R, Kamphuisen PW. Circulation 2008; 117: 93-102

Smoking increases the risk of venous thrombosis and acts synergistically with oral contraceptive use. Pomp ER, Rosendaal FR, Doggen CJM. Am J Hematol 2008; 83: 97-102

Risk of venous thrombosis: obesity and its joint effect with oral contraceptive use and prothrombotic mutations. Pomp ER, Le Cessie S, Rosendaal FR, Doggen CJM. Br J Haematol 2007; 139: 289-296

It has generally been held that the risk factors for arterial and venous thrombosis (VTE) are different, though in recent years it has been recognised that obesity is an important risk factor for both conditions. Three papers have recently been published from a systematic review/metaanalysis (Ageno et al., 2008) and a large case control study (Pomp et al., 2007, 2008), which suggest that smoking, hypertension and diabetes (as well as obesity) are significantly associated with VTE. Although the design of the case-control study has flaws, if anything, these might diminish the magnitude of the effects seen. The researchers found that smokers who did not take the pill were at twice the risk of VTE of non-smoking, non-pill users, while smokers who took the pill had eight times the risk [odds ratio (OR) $8.79,95 \% \mathrm{CI}$ 5.73-13.49], suggesting a synergistic relationship between the two. Women with a body mass index (BMI) $>30$ who took the pill had an OR of 23.78 (95\% CI 13.35-42.34) for VTE, while those with a BMI $>30$ who did not take the pill had an OR of 3.04 (95\% CI 1.66-5.57). Apart from the prescribing implications, the findings are of interest when looking at other studies of the pill and VTE, which have often not controlled for these factors. In addition, it highlights the potential for prescriber bias in studies prior to 1995 , since it was a widely held view that thirdgeneration pills would be safer for those with arterial risk factors. 1

Reviewed by Anne Szarewski, PhD, FFSRH Clinical Consultant and Honorary Senior Lecturer, Cancer Research UK Centre for Epidemiology, Mathematics and Statistics, Wolfson Institute of Preventive Medicine, London, UK

\section{Reference}

Szarewski A, Mansour D. The 'pill scare': responses of authorities, doctors, and patients using oral contraception. Hum Reprod patients using oral 
Ovarian cancer and oral contraceptives: collaborative reanalysis of data from 45 epidemiological studies including 23257 women with ovarian cancer and 87303 controls. Collaborative Group on Epidemiological Studies of Ovarian Cancer, Beral V, Doll R, Hermon C, Peto R, Reeves G. Lancet 2008; 371: 303-314

This is another of the large meta-analyses regularly produced by this group of researchers. This time they have looked at ovarian cancer risk and the combined oral contraceptive pill (COC), and confirmed that the COC greatly reduces the risk. The risk decreased by $20 \%$ with every 5 years of COC use, and for women who took the pill for 15 years, the risk was halved. The duration of protection lasted for many years after stopping the pill; even after 30 years there was still a significant reduction in risk [relative risk (RR) 0.86 , 95\% CI 0.76-0.97]. Between 10 and 19 years after stopping, the RR was 0.67 (95\% CI $0.62-0.73$ ), namely a roughly $40 \%$ reduction in risk. Importantly, the authors conclude that the protective effect is similar for both high- and lowestrogen dose pills. They estimate that around 200000 ovarian cancers have already been prevented by COC use in the last 50 years and predict that around 30000 cases of ovarian cancer per year will be prevented in future. Two accompanying editorials both suggest that the pill should be made available over the counter, though without any suggestions of how this should be done in practice to maintain patient safety.

Reviewed by Anne Szarewski, PhD, FFSRH Clinical Consultant and Honorary Senior Lecturer, Cancer Research UK Centre for Epidemiology, Mathematics and Statistics, Wolfson Institute of Preventive Medicine, London, UK

\section{LETTERS TO THE EDITOR}

\section{Difficult insertion of IUS}

We were relieved to read the letter ${ }^{1}$ about difficult insertion of the intrauterine system (IUS) in the October 2007 issue of the Journal because we and at least one other colleague have had exactly the same experience.

I (MD) have been fitting intrauterine devices (IUDs) for over 35 years and have had six or seven of these in the last year, each needing another IUS or indeed another IUD usually the TT380 Slimline ${ }^{\circledR}$. I fit on average 40 IUDs per year.

A colleague, who is also a general practitioner, with more than 5 years' experience, fits on average 20 per year. She has come across this problem twice, one episode requiring opening a third IUS to get it fitted, thus believing it must have been her own error (despite fitting it in the same way as always).

Finally, a locum doctor, of many years experience as myself, had one.

I have retained two of these devices, whose batch numbers are different.

We are now concerned that there is something wrong with the technique so that there may also be devices not correctly placed at the fundus.

The fitting of the TT380 Slimline differs entirely as the plunger is held at the base of the IUD before removing the insertion tube so we have never had a problem with it.

Jeanette Ledbury, FFSRH

Clinical Medical Officer, Saltergate

Contraception and Sexual Health, Saltergate

Health Centre, Chesterfield, UK

Margaret Duncan, MRCGP, DFSRH

General Practitioner and Staff Grade Doctor,

Saltergate Contraception and Sexual Health,

Saltergate Health Centre, Chesterfield, UK.

E-mail:margoduncan@mac.com

Reference

Albertazzi P. Difficult insertion of IUS [Letter]. J Fam Plann Reprod Health Care 2007; 33: 287.

\section{Reply}

With reference to the letter to the editor by $\mathrm{Dr}$ Albertazzi ${ }^{1}$ describing a case of difficult insertion of an intrauterine system (IUS), and the followup letter by Drs Ledbury and Duncan, ${ }^{2}$ we would like to take the opportunity to respond to these letters.

Bayer Schering Pharma considers customer feedback to be an extremely valuable tool in continuing to develop products that deliver the highest levels of customer satisfaction as well as to ensure their safe use. Spontaneous feedback is particularly important in order to make us aware of potential problems, enabling us to deal with them in a prompt and appropriate manner.

To enable thorough evaluation of individual cases, it is important that all available material (in the case of IUSs, the IUS with threads, the inserter and the outer package containing the batch number) together with a description of the difficulty encountered is forwarded for investigation. Details of how this can be arranged should be discussed with the local subsidiary of the company.

In the case described by Dr Albertazzi, ${ }^{1}$ a quality defect could be suspected, based on the failure of the IUS to deploy after two consecutive attempts. An attempt with a new IUS and inserte was subsequently successful. It should be noted that without the sample, a detailed evaluation cannot be made and therefore the possibility of a manufacturing defect in this specific case cannot be assessed. However, such a manufacturing defect remains a possibility.

The insertion technique of Mirena ${ }^{\circledR}$ is unique, and therefore, all health care professionals are encouraged to become fully familiar with the insertion instructions provided in each Mirena package, and to follow them in detail to ensure correct deployment and placement of the IUS. We have previously identified handling errors that can lead to a nondeployment of the IUS. In addition to the insertion instructions provided in the Mirena package, further material and advice, as well as demonstration systems (demonstration inserter with IUS and uterus models), training and support can be obtained via the local sales representatives or upon written request to the local subsidiary.

\section{Jussi Pirjola}

Director, Quality Management, Bayer Schering Pharma Oy, The Netherlands

\section{Sarah Cross}

Head of Medical Women's Health, Bayer Schering Pharma, Bayer House, Strawberry Hill, Newbury RG14 1JA, UK

\section{References \\ Albertazzi P. Difficult insertion of IUS [Letter]. J Fam Plann Reprod Health Care 2007; 33: 287. \\ Ledbury J, Duncan M. Difficult insertion of IUS [Letter].} $J$ Fam Plann Reprod Health Care 2008; 34: 134.

\section{LNG IUS duration of use}

I think the readership of the Journal would be interested in a discussion on intrauterine contraception held at an international meeting in New York, USA in November 2006. The presentations have since been published in a special supplement of Contraception in 2007 but the question and answer sessions remain unreported. ${ }^{1}$ During this meeting a question was asked concerning the duration of use for the levonorgestrel intrauterine system (LNG IUS) Irvin Sivin and Viveca Odlind were in the audience [authors on several papers reporting randomised controlled trial (RCT) findings that the LNG IUS provides effective contraception for up to 7 years].2,3 It was confirmed that all these RCTs took place in the 1980s using a LNG IUS prototype. This system released about $20 \mu \mathrm{g}$ LNG a day (similar to Mirena ${ }^{\circledR}$, Bayer Schering Pharma) but contained $60 \mathrm{mg}$ rather than $52 \mathrm{mg}$ LNG in the vertical stem. Therefore we need better data investigating the marketed system, Mirena, before concluding that this IUS is effective for 7 years.

Diana Mansour, FRCOG, FFSRH

Consultant in Community Gynaecology, Contraception and Sexual Health Service, Newcastle upon Tyne Primary Care Trust, Graingerville Clinic, Newcastle upon Tyne, UK. E-mail: Diana.Mansour@newcastle-pct.nhs.uk

\section{References}

Special issue on intrauterine devices and systems. Contraception 2007; 75: S1-S166.

2 Sivin I, Stern J, Coutinho E, Mattos CE, el Mahgoub S, Diaz S, et al. Prolonged intrauterine contraception: a seven-year randomized study of the levonorgestrel 20 $\mathrm{mcg} /$ day $(\mathrm{LNg} \mathrm{20})$ and the Copper T380 Ag IUDS Contraception 1991; 44: 473-480.

3 Barbosa I, Olsson SE, Odlind V, Goncalves T, Coutinho E. Ovarian function after seven years' use of a levonorgestrel IUD. Adv Contracept 1995; 11: 85-95.

\section{Difficult IUD insertions}

I write in response to the issue raised by Dr Isabel Draper. ${ }^{1}$ I share her sentiments that insertion of intrauterine contraception has become progressively more challenging as we see increasing numbers of nulliparous women requesting a copper intrauterine device (IUD) or Mirena ${ }^{\circledR}$ for contraception plus many older women requesting a Mirena ${ }^{\circledR}$ for gynaecological indications and for hormone replacement therapy. In the community clinic setting, we may have eight or nine such women for intrauterine contraception in a session plus have to balance this with the needs of a training doctor.

Insertion of intrauterine contraception is often deeply unpleasant for nulliparous and older women, particularly if the procedure is being undertaken by an inexpert doctor. A carefully and gently applied intracervical injection of local anaesthetic makes a huge difference to the tolerability of difficult and painful insertions. Local anaesthetic allows for easier insertion of the banded copper IUD or Mirena. In addition, local anaesthetic blocks the vasovagal response which can have an impact on the smooth running of a busy clinic when nulliparous women may languish feeling faint and in pain following IUD insertion without local anaesthetic. In the training situation, if the instructing doctor first inserts the local anaesthetic then the training doctor can then proceed with the IUD or Mirena insertion in a much less stressful situation.

With this in mind, I asked a nulliparous woman last week following her second IUD insertion which was better: with or without local anaesthetic? She said that it was " a thousand times better" with local anaesthetic.

Ailsa E Gebbie, FRCOG, FFSRH

Consultant in Community Gynaecology, NHS Lothian, Edinburgh, UK.

E-mail:ailsa.gebbie@nhslothian.scot.nhs.uk

Reference

Draper IB. Difficult IUD insertions [Letter]. J Fam Plann Reprod Health Care 2008; 34: 67. 